\title{
Diquark Condensation in High Density Baryon Matter
}

\author{
Thomas Schäfer ${ }^{\mathrm{a}}$ \\ anstitute for Nuclear Theory \\ University of Washington \\ Seattle, WA 98195
}

We argue that cold quark matter is a diquark Bose condensate. The Cooper pairs of QCD are spin-isospin zero, color anti-symmetric quark pairs. For two light flavors, instanton effects lead to gaps on the order of $50 \mathrm{MeV}$.

\section{Introduction}

Over the past years, important progress has been achieved in our understanding of the phase structure of QCD at finite temperature. Even though many important points remain to be worked out (like the nature of the QCD phase transition for realistic values of the quark masses), there is a nice frame work based on universality arguments and our ability to perform simulations on the lattice.

For reasons that we do not need to reiterate here, the problem of cold dense matter is much less understood. On the other hand, it has been realized for quite some time that the possible phase structure of dense matter is very rich. In addition to the nuclear and quark matter phases, new phases containing pion or kaon condensates, strange quark matter, etc., have been suggested. In this contribution we want to study the possibility that cold quark matter is in a superconducting phase. The Cooper pairs of QCD are spin zero diquarks.

Unlike many of the phases that we just mentioned, this phenomenon is very robust and independent of the detailed dynamics. It is based on the observation that a sharp Fermi surface is expected to be unstable with respect to pair condensation whenever there is an (arbitrarily weak!) attractive interaction between quarks pairs in the vicinity of the Fermi surface.

\section{The Quark-Quark interaction}

While the phenomenon as such is independent of the strength and the exact form of the interaction, the size of the gap, the condensation energy, the critical temperature etc., certainly depend on the interaction. If the chemical potential is very large, we expect the interaction to be perturbative. The Coulomb interaction between quarks is attractive if the two quarks are in a spin-isospin zero, color anti-triplet state. Color superconductivity induced by perturbative gluon exchange was first studied in detail by Bailin and Love [1] (the possibility of superconductivity in cold quark matter was apparently first pointed 

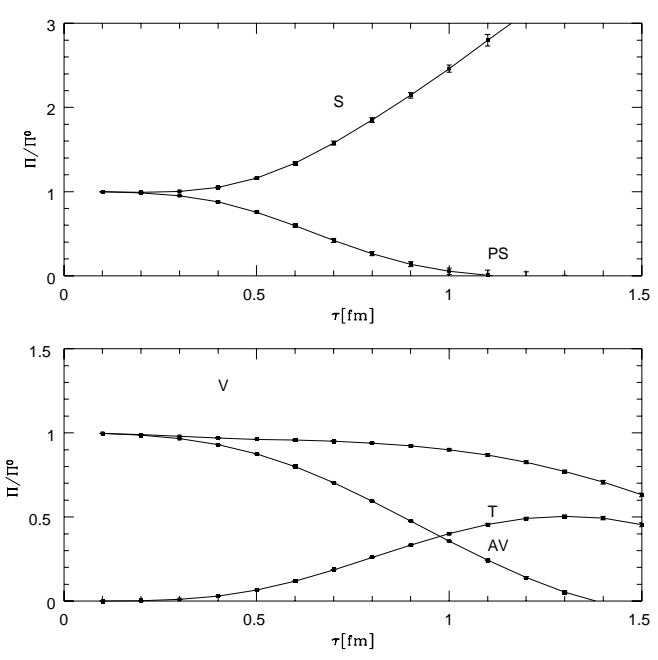

Figure 1. Color $\overline{3}$ diquark correlation functions.
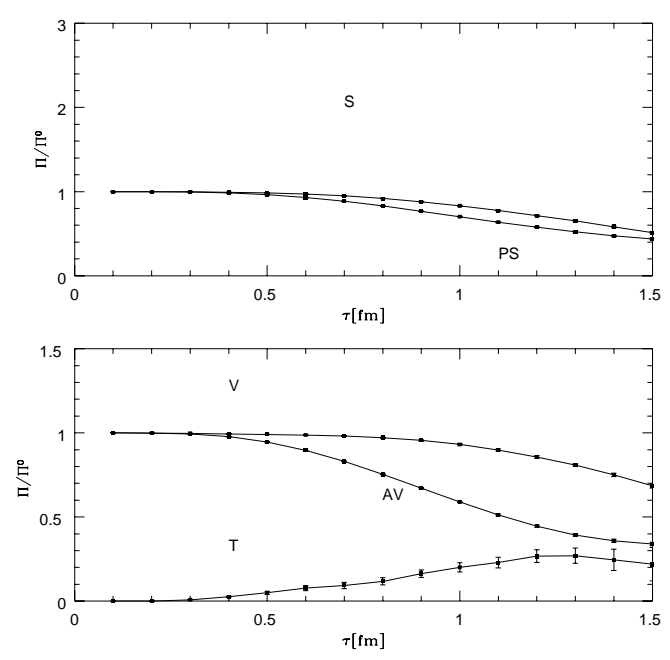

Figure 2. Color 6 diquark correlation functions.

out by Frautschi [2]). These authors find that at baryon densities $\rho \sim(5-10) \rho_{0}$, where $\rho_{0}$ is nuclear matter density, both the gap and the critical temperature are on the order of $1 \mathrm{MeV}$.

In this contribution we show that non-perturbative effects can lead to diquark condensates with $\Delta, T_{c}$ about two orders of magnitude bigger [3, 4]. These non-perturbative effects are connected with instantons. For two flavors (up and down) the $(\bar{q} q)$ interaction generated by instantons is given by [5]

$\mathcal{L}=G \frac{1}{4\left(N_{c}^{2}-1\right)}\left\{\frac{2 N_{c}-1}{2 N_{c}}\left[\left(\bar{\psi} \tau_{\alpha}^{-} \psi\right)^{2}+\left(\bar{\psi} \gamma_{5} \tau_{\alpha}^{-} \psi\right)^{2}\right]+\frac{1}{4 N_{c}}\left(\bar{\psi} \sigma_{\mu \nu} \tau_{\alpha}^{-} \psi\right)^{2}\right\}$

where $N_{c}$ is the number of colors and $\tau^{-}=(\vec{\tau}, i)$ is an isospin matrix. Phenomenology (or interacting instanton calculations) gives $G \simeq 490 \mathrm{GeV}^{-2}$. There is a great deal of evidence that this simple interaction correctly describes many aspects of hadronic phenomenology, like chiral symmetry breaking and correlation functions of hadronic currents. See [6] for a review of these issues.

The result (11) can be Fierz-rearranged into a $(q q)$ interaction. We find

$$
\begin{aligned}
\mathcal{L} & =G\left\{-\frac{1}{16 N_{c}\left(N_{c}-1\right)}\left[\left(\psi^{T} C \tau_{2} \lambda_{A}^{a} \psi\right)\left(\bar{\psi} \tau_{2} \lambda_{A}^{a} C \bar{\psi}^{T}\right)+\left(\psi^{T} C \tau_{2} \lambda_{A}^{a} \gamma_{5} \psi\right)\left(\bar{\psi} \tau_{2} \lambda_{A}^{a} \gamma_{5} C \bar{\psi}^{T}\right)\right]\right. \\
& \left.+\frac{1}{32 N_{c}\left(N_{c}+1\right)}\left(\psi^{T} C \tau_{2} \lambda_{S}^{a} \sigma_{\mu \nu} \psi\right)\left(\bar{\psi} \tau_{2} \lambda_{S}^{a} \sigma_{\mu \nu} C \bar{\psi}^{T}\right)\right\}
\end{aligned}
$$

Here, $C$ is the charge conjugation matrix, $\tau_{2}$ is the anti-symmetric Pauli matrix, $\lambda_{A, S}$ are the anti-symmetric (color $\overline{3}$ ) and symmetric (color 6 ) color generators. The effective lagrangian (2) provides a strong attractive interaction between an up and a down quark with anti-parallel spins $\left(J^{P}=0^{+}\right)$in the color anti-triplet channel, and a repulsive interaction in the $0^{-}$channel. 
The scalar diquark current $j_{S}^{a}=\epsilon_{a b c} q_{b}^{T} C \gamma \tau_{2} q_{c}$ is of course not gauge invariant, and does not couple to physical states. But we can neutralize color by adding an infinitely heavy quark, and consider correlation functions of the gauge invariant current $j=j_{S}^{a} Q^{a}$. In the limit $m_{Q} \rightarrow \infty$, the propagator of the heavy quark reduces to a gauge string.

The instanton liquid does not confine, and we can calculate the mass of two-quark bound states. From a simple RPA calculation, we find a scalar diquark mass of $m_{S} \simeq 400$ $\mathrm{MeV}$, which is significantly below the two-quark threshold, $2 m_{q}-m_{S} \simeq 200-300 \mathrm{MeV}$. All other channels (vectors and axial-vectors, color 6 diquarks, etc.) are at most very weakly bound. This result is consistent with earlier calculations in the NJL model, see for example [7]. A calculation of diquark masses in the instanton model was also performed in 88. These authors perform a simultaneous meson-diquark bosonization of the interaction (1). In their scheme, only a fraction $1 / N_{c}$ of the interaction acts in the diquark channel. As a result, the scalar diquark is unbound.

Higher order effects can be taken into account by performing numerical calculations of diquark correlation functions in the instanton liquid [9]. Results for scalar (S), pseudoscalar (PS), vector $(\mathrm{V})$, axial-vector $(\mathrm{AV})$, and tensor $(\mathrm{T})$ correlation functions, in both color $\overline{3}$ and 6 channels are shown in Figs. 1 and 2. We observe that only the color $\overline{3}$ scalar shows substantial attraction. Numerically, we find $m_{S} \simeq 400 \mathrm{MeV}$, and a scalar-vector diquark splitting of almost $500 \mathrm{MeV}$. Recently, Heß et al. calculated diquark correlation functions (in a fixed gauge, not with the gauge string included) on the lattice $[10$. They find $m_{S} \simeq 650 \mathrm{MeV}$ and a smaller scalar-vector diquark splitting, $m_{V}-m_{S}=100$ $\mathrm{MeV}$. On the other hand, they also have a nucleon-delta splitting which is too small, $m_{\Delta}-m_{N}=150 \mathrm{MeV}$, while the instanton model tends to overestimate this quantity. So the truth is probably somewhere in between.

The fact that color $\overline{3}$ scalar-isoscalar diquarks are favored is not specific to instantons. If the chemical potential is very large, semi-classical fields are exponentially suppressed, and the quark-quark interaction is dominated by one-gluon exchange. The effective fourfermion vertex corresponding to one-gluon exchange is

$$
\begin{aligned}
\mathcal{L}_{O G E}= & \frac{G_{V}}{2}\left\{\frac{4}{3}\left[\left(\psi^{T} C \gamma_{5} \tau_{2} \lambda_{A}^{a} \psi\right)\left(\bar{\psi} \gamma_{5} \tau_{2} \lambda_{A}^{a} C \bar{\psi}^{T}\right)-\left(\psi^{T} C \tau_{2} \lambda_{A}^{a} \psi\right)\left(\bar{\psi} \tau_{2} \lambda_{A}^{a} C \bar{\psi}^{T}\right)\right]\right. \\
& \left.+\frac{2}{3}\left[\left(\psi^{T} C \gamma_{\mu} \gamma_{5} \tau_{2} \lambda_{A}^{a} \psi\right)\left(\bar{\psi} \gamma_{\mu} \gamma_{5} \tau_{2} \lambda_{A}^{a} C \bar{\psi}^{T}\right)+\left(\psi^{T} C \gamma_{\mu} \tau_{2} \vec{\tau} \lambda_{A}^{a} \psi\right)\left(\bar{\psi} \gamma_{\mu} \tau_{2} \vec{\tau} \lambda_{A}^{a} C \bar{\psi}^{T}\right)\right]\right\},
\end{aligned}
$$

which is also attractive in the scalar diquark channel. The coupling is given by $G_{V}=$ $\left(4 \pi \alpha_{s}\right) / \Lambda^{2}$, where $\Lambda$ is some IR cutoff. In the high density phase, it seems reasonable to set $\Lambda$ equal to the Debye mass.

In the vicinity of the chiral phase transition, higher order instanton effects may also play a role. The interaction induced by instanton-anti-instanton molecules is [11

$$
\begin{aligned}
\mathcal{L}_{I A}= & G_{M}\left\{\frac{1}{6}\left[\left(\psi^{T} C \gamma_{5} \tau_{2} \lambda_{A}^{a} \psi\right)\left(\bar{\psi} \gamma_{5} \tau_{2} \lambda_{A}^{a} C \bar{\psi}^{T}\right)-\left(\psi^{T} C \tau_{2} \lambda_{A}^{a} \psi\right)\left(\bar{\psi} \tau_{2} \lambda_{A}^{a} C \bar{\psi}^{T}\right)\right]\right. \\
& \left.+\frac{1}{24}\left[\left(\psi^{T} C \gamma_{\mu} \gamma_{5} \tau_{2} \lambda_{A}^{a} \psi\right)\left(\bar{\psi} \gamma_{\mu} \gamma_{5} \tau_{2} \lambda_{A}^{a} C \bar{\psi}^{T}\right)-\left(\psi^{T} C \gamma_{\mu} \tau_{2} \vec{\tau} \lambda_{A}^{a} \psi\right)\left(\bar{\psi} \gamma_{\mu} \tau_{2} \vec{\tau} \lambda_{A}^{a} C \bar{\psi} \bar{\psi}^{T}\right)\right]\right\},
\end{aligned}
$$

which is also most attractive in the scalar diquark channel. 


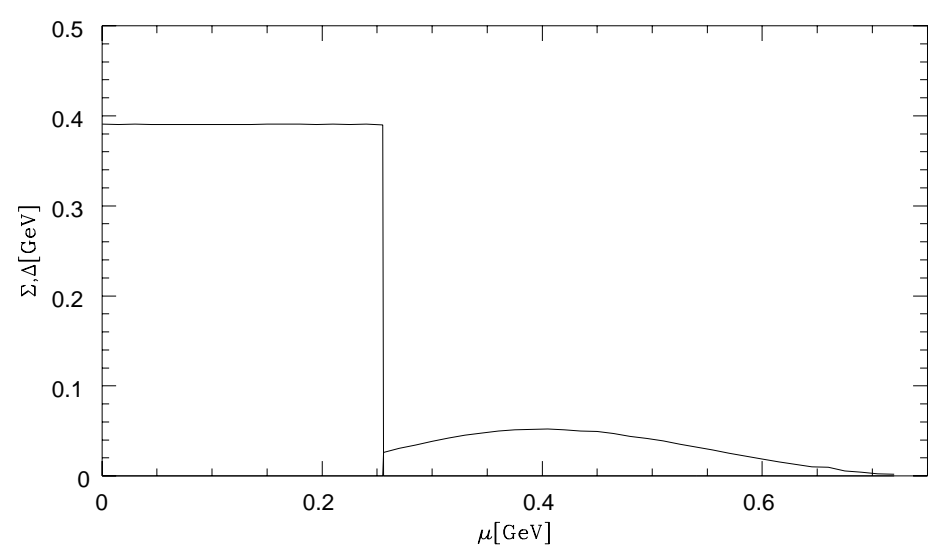

Figure 3. Chiral and diquark gaps $\Sigma$ and $\Delta$ as a function of the quark chemical potential.

\section{Diquark Condensation}

We now turn to a simple mean field model of chiral symmetry breaking and diquark condensation. We consider the instanton induced effective interaction (II) and a trial state with both quark $\langle\bar{q} q\rangle$ and diquark $\left\langle q^{T} C \gamma_{5} \lambda_{2} \tau_{2} q\right\rangle$ condensates. We will denote the corresponding gaps by $\Sigma$ and $\Delta$.

At small chemical potential, quark-anti-quark condensation is favored over diquark condensation. The size of the gap is controlled by the standard, NJL-type gap equation. A chiral condensate is only formed if the interaction strength exceeds a certain critical value. If the chemical potential is increased, the quark-anti-quark interaction is partially blocked, but the quark-quark interaction in the vicinity of the Fermi surface is enhanced. The diquark gap equation in the high density phase is

$1=\frac{8}{(2 \pi)^{2}} G(\mu) \int p^{2} d p \frac{F\left(\left(p-p_{F}\right)^{2}\right)}{(p-\mu)^{2}+\Delta^{2}}$,

which shows the logarithmic enhancement near $p=p_{F}$. In the high density phase, the coupling is exponentially suppressed, $G(\mu)=G(0) \exp \left[-N_{f} \rho^{2} \mu^{2} \theta\left(\mu-\mu_{c}\right)\right]$. The quark interaction is due to instanton zero modes near the Fermi surface. The corresponding form factor $F$ is therefore peaked at $p_{F}$. Here, we assume a simple monopole shape with a range $\Lambda=300 \mathrm{MeV}$. The result for the coupled system of gap equations is shown in Fig. 3. At $\mu=0$ the chiral gap is $\Sigma=400 \mathrm{MeV}$ (this is how we fixed $G(0)$ ). We then find a first order transition to a diquark condensed state at $\mu=270 \mathrm{MeV}$ (see also [12]). The gap first grows because of the increase in the number of states near the Fermi surface, but becomes very small at large chemical potential because of the instanton suppression factor. The maximum gap $\Delta$ is on the order of $50 \mathrm{MeV}$.

It is instructive to discuss the structure of the diquark condensed phase in terms of the corresponding Landau-Ginzburg effective free energy

$F=\alpha\left|\left(\Delta^{a}\right)^{2}\right|+\beta\left|\left(\Delta^{a}\right)^{2}\right|^{2}+\gamma\left|\left(D_{\mu} \Delta\right)^{2}\right|+\frac{1}{4} G_{i j}^{2}+\ldots$ 

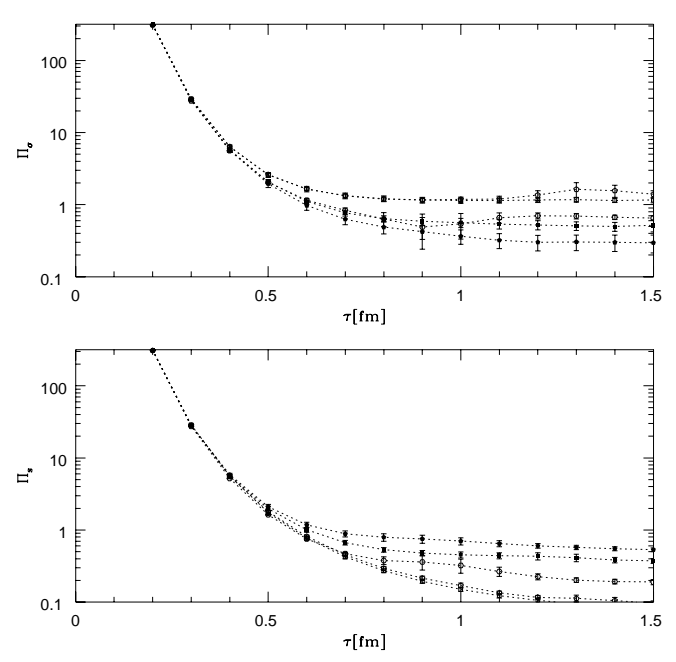

Figure 4. Scalar $(\bar{q} q)$ and $\left(q^{T} C \gamma_{5} q\right)$ correlation functions for different chemical potentials.
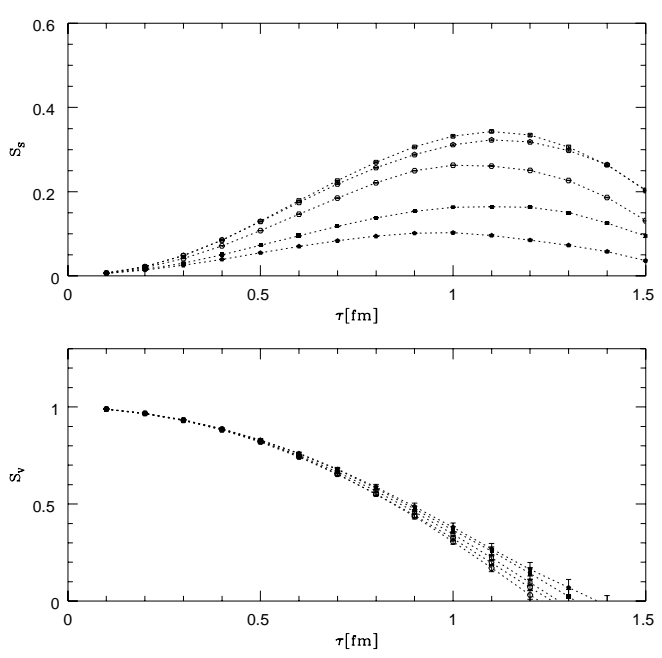

Figure 5. Scalar $\operatorname{Tr}(S)$ and vector $\operatorname{Tr}\left(\gamma_{0} S\right)$ components of the quark propagator for different chemical potentials.

The coefficients $\alpha$ and $\beta$ can be matched to the gap equation (2), while $\gamma$ can be determined by performing a derivative expansion. From the effective action, we can read off the condensation energy! $\epsilon=-30 \mathrm{MeV} / \mathrm{fm}^{3}$ and the coherence length $\xi_{\Delta}=(\gamma / \alpha)^{1 / 2} \simeq 0.8$ fm. Among the eight gluons, 5 get a mass via the Higgs mechanism (corresponding to breaking color $S U(3)$ to $S U(2))$. The mass of the off-diagonal gluons is $m_{g}=(\gamma / 2)^{1 / 2} g \Delta$. From this, we get a penetration depth $\xi_{A} \simeq 1.5 \mathrm{fm}$. The order parameter carries electric charge, but the diagonal gluons mix with the photon, producing a new massless gauge boson. The corresponding Weinberg angle is small, $\tan \theta=e /(\sqrt{3} g)$.

It is unfortunate that, at least in the case of two flavors, the effective action does not support any textures. We should note that, since the condensation energy is small, the critical color-magnetic field is also small, $B^{2} \simeq(130 \mathrm{MeV})^{2}$. This is significantly smaller than the field inside an instanton, which implies that the order parameter is probably very inhomogeneous.

\section{QCD with two colors}

A nice model system in which we can study diquark condensation is QCD with two colors. $N_{c}=2$ QCD has a particle-anti-particle (Pauli-Gürsey) symmetry. This symmetry implies that mesons and diquarks are degenerate, and that (in the limit of zero mass) the quark-anti-quark condensed state is equivalent to a diquark condensed state. If the quark mass is finite, the degeneracy is lifted and the true ground-state has a chiral condensate. If we now also turn on a chemical potential, we expect the order parameter to rotate in the diquark direction as soon as $\mu$ exceeds a critical value on the order of scalar diquark

\footnotetext{
${ }^{1}$ This sounds small, but it is not so different from the chiral condensation energy $\epsilon \simeq-f_{\pi}^{2} \Sigma^{2} / 2 \simeq$ $-75 \mathrm{MeV} / \mathrm{fm}^{3}$.
} 
mass.

This phenomenon can be studied in imaginary time simulations, because the fermion determinant remains real even in the presence of a chemical potential. As an example, we consider the instanton liquid simulations described in [13]. The chiral condensate is easy to measure, and we observe the expected drop at large $\mu$. The diquark condensate cannot be studied directly, but we can measure the diquark correlation function. If diquarks are condensed, the correlator will tend to a finite value at large distance. This is indeed observed in the results shown in Fig. 4. (In the case of the diquark correlator, the lower points correspond to small $\mu$, the upper points to large $\mu$. For the $(\bar{q} q)$ correlator, the situation is reversed.)

We can also study the quark propagator in more detail. The scalar (chirality violating) component disappears as the chemical potential is increased, see Fig. 5. But the vector part changes very little, indicating that the gap in the spectrum remains, even though the quark condensate goes to zero.

\section{Summary}

Straightforward arguments suggest that cold quark matter is a superconductor. Instanton effects can lead to sizeable gaps, and we estimate the maximum gap to be on the order of $50 \mathrm{MeV}$. The phase structure of the condensed state, in particular if strange quarks are included, is very rich, and many interesting phenomena remain to be explored.

\section{REFERENCES}

1. D. Bailin and A. Love, Phys. Rep. 107, 325 (1984).

2. S. C. Frautschi, Asymptotic freedom and color superconductivity in dense quark matter, in: Proceedings of the Workshop on Hadronic Matter at Extreme Energy Density, N. Cabibbo, Editor, Erice, Italy (1978)

3. M. Alford, K. Rajagopal and F. Wilczek, hep-ph/9711395, Phys. Lett. B422, 247 (1998)

4. R. Rapp, T. Schäfer, E. Shuryak, M. Velkovsky, hep-ph/9711396, Phys. Rev. Lett., in press

5. G. 't Hooft, Phys. Rev. D14, 3432 (1976).

6. T. Schäfer and E.V. Shuryak, Instantons in QCD, hep/ph 9610451, Rev. Mod. Phys. 70, 323 (1998)

7. U. Vogl, Z. Phys. A337, 191 (1990)

8. D. Diakonov, H. Forkel, M. Lutz, Phys. Lett. B373, 147 (1996)

9. T. Schäfer, E.V. Shuryak, J.J.M. Verbaarschot, Nucl. Phys. B412, 143 (1994)

10. M. Hess, F. Karsch, E. Laermann, I. Wetzorke, preprint, hep-lat/9804023

11. T. Schäfer, E.V. Shuryak, J.J.M. Verbaarschot, Phys. Rev. D51, 1267 (1995)

12. J. Berges, K. Rajagopal, preprint, hep-ph/9804233

13. T. Schäfer, hep-ph/9708256, Phys. Rev. D57, 3950 (1998) 